\title{
Generalized ordering-search for learning directed probabilistic logical models
}

\author{
Jan Ramon • Tom Croonenborghs • Daan Fierens • \\ Hendrik Blockeel • Maurice Bruynooghe
}

Received: 1 October 2007 / Accepted: 9 October 2007 / Published online: 19 December 2007

Springer Science+Business Media, LLC 2007

\begin{abstract}
Recently, there has been an increasing interest in directed probabilistic logical models and a variety of formalisms for describing such models has been proposed. Although many authors provide high-level arguments to show that in principle models in their formalism can be learned from data, most of the proposed learning algorithms have not yet been studied in detail. We introduce an algorithm, generalized ordering-search, to learn both structure and conditional probability distributions (CPDs) of directed probabilistic logical models. The algorithm is based on the ordering-search algorithm for Bayesian networks. We use relational probability trees as a representation for the CPDs. We present experiments on a genetics domain, blocks world domains and the Cora dataset.
\end{abstract}

Keywords Bayesian networks · Probabilistic logical models · Ordering-search

\section{Introduction}

Probabilistic logical models have recently gained a lot of attention as tools to deal with problem domains with a complex, relational structure and stochastic or noisy characteristics. On the one hand there are undirected models that are relational extensions of Markov

Editors: Stephen Muggleton, Ramon Otero, Simon Colton.

J. Ramon $(\bowtie) \cdot$ T. Croonenborghs · D. Fierens · H. Blockeel · M. Bruynooghe

Dept. of Computer Science, K.U. Leuven, Celestijnenlaan 200A, 3001 Leuven, Belgium

e-mail: Jan.Ramon@cs.kuleuven.be

T. Croonenborghs

e-mail: Tom.Croonenborghs@cs.kuleuven.be

D. Fierens

e-mail: Daan.Fierens@cs.kuleuven.be

H. Blockeel

e-mail: Hendrik.Blockeel@cs.kuleuven.be

M. Bruynooghe

e-mail: Maurice.Bruynooghe@cs.kuleuven.be 
networks (Richardson and Domingos 2006) or dependency networks (Neville and Jensen 2004). On the other hand there are directed models that are usually relational extensions of Bayesian networks. One of the advantages of undirected models is their ability to deal with cyclic dependencies, a disadvantage is that this usually complicates semantics and inference. More precisely, inference for undirected models often relies on Markov Chain Monte Carlo approaches such as Gibbs sampling. Such approaches are computationally expensive and need special care when applied on models with deterministic dependencies as often occur in background knowledge of ILP applications. Hence, in this paper we focus on directed probabilistic logical models.

A variety of formalisms for describing directed probabilistic logical models has been proposed: Probabilistic Relational Models (Getoor et al. 2001), Bayesian Logic Programs (Kersting and De Raedt 2001), Logical Bayesian Networks (Fierens et al. 2005a) and many others. Although most authors describe high-level algorithms to learn models in their formalism or provide arguments to show that such algorithms can be developed, there are still many problems that have not been studied in detail. One such problem is how to deal with recursive dependencies. Consider for example the blocks world (Slaney and Thiébaux 2001), where we have a set of blocks that can be stacked on top of each other. We can use the on $/ 2$ predicate to represent a configuration of blocks: for each pair $(A, B)$ of blocks we have to state whether on $(A, B)$ is true or false. Obviously some (recursive) dependencies hold between all the different $o n / 2$ facts. There are some simple, deterministic dependencies (e.g. if $o n(a, b)$ is true than $o n(b, a)$ is false) but there might also be some more complex, probabilistic dependencies (e.g. stacks of many blocks might be unlikely due to instability). In undirected models we could state that each on $/ 2$ fact depends on all other on $/ 2$ facts, but in directed models this is not allowed since it would lead to cycles. Hence, learning a directed probabilistic logical model of the dependencies between all on $/ 2$ facts is a challenging problem.

In this paper we introduce an algorithm, generalized ordering-search, to learn both structure and conditional probability distributions of directed probabilistic logical models. Our algorithm is based on the ordering-search algorithm of Teyssier and Koller (2005) for learning propositional Bayesian networks. Our contribution is that we upgrade this algorithm to the relational case and investigate the use of relational probability trees (Neville et al. 2003; Fierens et al. 2005b) as compact and interpretable models of conditional probability distributions.

The remainder of this paper is structured as follows. In Sect. 2 we review learning algorithms for Bayesian networks. In Sect. 3 we introduce our algorithm for learning directed probabilistic logical models. In Sect. 4 we validate our work through a set of experiments. In Sect. 5 we conclude and provide some directions for further work.

\section{The propositional case: learning Bayesian networks}

Recall that a Bayesian network is a compact specification of a joint probability distribution on a set of random variables (RVs) under the form of a directed acyclic graph (the 'structure') and a set of conditional probability distributions (CPDs) (Neapolitan 2003). There exist several approaches for learning Bayesian networks. We now review two approaches, structure-search and ordering-search, since we need the introduced terminology later.

The most traditional approach to learning Bayesian networks is to perform structuresearch (Heckerman et al. 1995), which is basically hill climbing through the space of possible structures. Starting from an initial structure, a number of possible refinements of this 
structure are evaluated and the best refinement is chosen as the new structure. This process is repeated until an accurate structure is found or until none of the refinements yields any improvement. A refinement of a structure is usually obtained by adding, deleting or reversing an edge in the current structure. Only refinements that are acyclic graphs are allowed (hence for each refinement an acyclicity check needs to be performed). To assess the quality of a structure a scoring criterion is used such as likelihood, marginal likelihood or minimum description length. Computing the score of a structure usually requires computing the optimal CPDs for the structure first.

A recent alternative approach is based on the observation that it is relatively easy to learn a Bayesian network if a causal ordering on the RVs is given (Teyssier and Koller 2005). More precisely, an ordering on the RVs eliminates the possibility of cycles and hence makes it possible to decide for each RV $X$ separately which RVs (from all RVs preceding $X$ in the ordering) are its parents. Suppose for instance that we use probability trees to represent CPDs. For each RV $X$ we learn a probability tree with as inputs all RVs preceding $X$ in the ordering. The probability tree identifies which of these RVs are relevant for predicting $X$ (they will become the parents of $X$ ) and specifies how they influence $X$ (the CPD for $X$ ). Hence, such a set of probability trees fully determines a Bayesian network.

In theory we can use any ordering on the RVs to learn a Bayesian network. ${ }^{1}$ However, the ordering used influences the compactness (number of edges) of the resulting Bayesian network. Some orderings might lead to a Bayesian network with only a few edges, while other orderings lead to an almost fully-connected network. This of course also influences learnability since a network with many edges has a lot of parameters and, as a consequence, is difficult to learn reliably from a limited amount of data. Hence, when using the above strategy to learn a Bayesian network it is important to use a good causal ordering. Unfortunately, such an ordering is usually not known in advance.

To solve the problem that a good ordering is usually not known, Teyssier and Koller (2005) introduced ordering-search. Ordering-search performs hill-climbing through the space of possible orderings, in each step applying the above strategy to learn a Bayesian network for the current ordering. A refinement of an ordering is obtained by swapping two adjacent RVs in the current ordering. The score of an ordering is defined as the score of the Bayesian network obtained for that ordering.

Teyssier and Koller (2005) experimentally compared structure-search and orderingsearch and found that ordering-search is always at least as good as structure-search and usually faster. As an explanation of these results, Teyssier and Koller note that the search space of orderings is smaller than the search space of structures and that ordering-search does not need acyclicity tests, which are costly if there are many RVs.

\section{The relational case: learning directed probabilistic logical models}

In this section we introduce an algorithm, generalized ordering-search, for learning directed probabilistic logical models. This algorithm is the result of upgrading the propositional ordering-search algorithm for Bayesian networks to the relational case. We start by giving some background on directed probabilistic logical models in Sect. 3.1. In Sect. 3.2 we

\footnotetext{
${ }^{1}$ This is because a joint probability distribution can be decomposed using any ordering. For example $P(X, Y, Z)$ can be decomposed as $P(X) P(Y \mid X) P(Z \mid X, Y)$ using the ordering $X, Y, Z$ or as $P(Z) P(Y \mid Z) P(X \mid Y, Z)$ using the ordering $Z, Y, X$, and so on.
} 
motivate why the propositional ordering-search algorithm needs to be upgraded and we explain our generalized ordering-search algorithm on a high level. In Sects. 3.3 and 3.4 we explain the different steps of the algorithm in more detail. For the sake of clarity, in Sect. 3.5 we give a brief overview of the entire algorithm. Finally, in Sect. 3.6 we discuss the relation with some other existing algorithms for learning directed probabilistic logical models.

\subsection{Directed probabilistic logical models}

We will use the terminology of Logical Bayesian Networks (Fierens et al. 2005a) but our discussion applies equally well to related probabilistic logical formalisms such as Bayesian Logic Programs (Kersting and De Raedt 2001) and Probabilistic Relational Models (Getoor et al. 2001).

A Logical Bayesian Network (LBN) is essentially a specification of a Bayesian network conditioned on some predicates describing the domain of discourse. An LBN consists of three parts: a set of random variable declarations specifying the RVs, a set of conditional dependency clauses specifying dependencies between these RVs and a set of CPDs quantifying these dependencies. We now illustrate this with two examples.

Example 1 (Genetics domain) Let us first consider a simple example concerning the inheritance of genes (De Raedt and Kersting 2004). We have a set of people with their family relationships; each person is characterized by having or not having a particular gene. Whether a person has that gene depends on the presence of the gene in the parents. Assuming there is no uncertainty about the family relationships, we can use standard logical predicates person $/ 1$ and parent $/ 2$ to model them. The uncertainty about the presence of the gene is modeled using a probabilistic predicate gene/1. Each ground atom gene $(p)$ represents an RV indicating whether person $p$ has the gene or not. The first part of the LBN states the relevant RVs, i.e. all gene $(P)$ RVs with $P$ a person:

$$
\operatorname{random}(\operatorname{gene}(P)) \leftarrow \operatorname{person}(P) \text {. }
$$

The second part of the $\mathrm{LBN}$ states the dependencies between the RVs, i.e. gene $(C)$ depends on gene $(P)$ if $P$ is a parent of $C$ :

$$
\text { gene }(C) \mid \text { gene }(P) \leftarrow \operatorname{parent}(P, C) \text {. }
$$

Finally, a full LBN for this example would also include a CPD for the gene $/ 2$ predicate that quantifies exactly how a gene $(C)$ depends on gene $(P)$.

Example 2 (Blocksworld domain) Let us now consider a more complex example from the blocks world. We have a set of blocks in a certain configuration and we know that certain dependencies hold between the positions of different blocks. To model this with an LBN we can use a logical predicate block/1 that identifies all blocks and a probabilistic predicate on $/ 2$ to model the configuration of the blocks. The relevant RVs are all on $(X, Y) \mathrm{RVs}$ with $X$ a block and $Y$ a block or the floor:

$$
\begin{aligned}
& \operatorname{random}(\text { on }(X, Y)) \leftarrow \operatorname{block}(X), \operatorname{block}(Y) . \\
& \operatorname{random}(\text { on }(X, \text { floor })) \leftarrow \operatorname{block}(X) .
\end{aligned}
$$


Next, we have to state the dependencies between the RVs. In the most general case we could assume some ordering on the RVs and specify that each RV can depend on all RVs preceding it in the ordering:

$$
\text { on }(W, X) \mid \text { on }(Y, Z) \leftarrow \text { ordering }(\text { on }(W, X) \text {, on }(Y, Z)) \text {. }
$$

Here ordering/2 is a logical predicate that specifies a (full or partial) ordering on ground on $/ 2$ atoms. The concrete ordering to be used depends on how the current configuration of blocks was created. For instance, if the configuration was created from some known previous configuration by applying some stochastic actions, the ordering could be determined by this previous configuration (see also Sect. 4 for more examples). Note that a full LBN for this example would also include a CPD for the on $/ 2$ predicate that quantifies how an on $(X, Y)$ $\mathrm{RV}$ depends on all RVs preceding it in the ordering. We will argue later that such CPDs can be represented by relational probability trees.

Usually for a particular application the RVs are given but the dependencies (including the definition of the ordering/2 predicate in the previous example) and the CPDs have to be learned. In the next sections we introduce a learning algorithm to do this.

\subsection{Generalized ordering-search: motivation and outline of the algorithm}

Directed probabilistic logical models, and in particular LBNs, can be learned from a set of examples each consisting of an interpretation of the logical predicates and a specification of all ground RVs and their values.

Since Teyssier and Koller (2005) found ordering-search to perform better than structuresearch for learning Bayesian networks, we will now generalize ordering-search to the relational case. As we discuss below, there are two major differences between the propositional case and the relational case which make the propositional ordering-search algorithm inapplicable to the relational case. This leads to two modifications to this algorithm.

\subsubsection{Difference 1: a model of the optimal ordering is needed}

First consider the case where the hypothesis space is restricted to LBNs that are acyclic at the predicate level. Obviously this does not include LBNs with recursive dependencies such as in our blocks world example. Generalizing ordering-search to such cases has not been done before but is rather straightforward. Instead of having an ordering on RVs like for Bayesian networks, we now need an ordering on the probabilistic predicates. We can perform hill-climbing over the space of predicate orderings in essentially the same way as over the space of RV orderings in the propositional case. As we explain later, the main difference with the propositional case is that now more general CPDs are needed.

Of course we also want to be able to learn LBNs that are cyclic at the predicate level (but acyclic at the ground level). An important class of such LBNs are LBNs with recursive dependencies such as in our blocks world example. For such cases the easy approach of having an ordering on the predicate level cannot be used anymore (since recursive dependencies would not be allowed then). Instead we need an ordering on ground RVs. The problem with this, however, is that the precise set of ground RVs depends on the domain of discourse (constants, ...) and we want to learn an LBN that generalizes over different domains of discourse. So we do not simply need an ordering on the ground RVs but a general model that given any domain of discourse determines an ordering on the ground RVs for that domain of discourse. 


\subsubsection{Difference 2: more general CPDs are needed}

One of the main differences between learning Bayesian networks and learning probabilistic logical models lies in the CPDs. In the relational case, we do not want to learn a separate CPD for each ground RV. We want a model that generalizes over different domains of discourse, which is usually obtained by doing some kind of "parameter sharing" across RVs built from the same predicate. In our case, this means that for each probabilistic predicate we want to learn a single function that can be used to generate the CPD for any ground RV built from that predicate. We call such a function a generalized conditional probability function $(G C P F)$. Informally, we can see a GCPF as a function that takes as input a target RV $X$ and the set of potential parents of $X$ and has as output the actual parents of $X$ and the precise CPD for $X$ with respect to these parents. In the next section we argue that such a GCPF can be represented and learned as a relational probability tree.

Two ground RVs from the same predicate might be at radically different positions in the ordering on ground RVs. Hence, the set of potential parents for these two RVs can be radically different. Consider again our blocks world example. The first $o n(X, Y) \mathrm{RV}$ in the ordering has no potential parents, while the last RV in the ordering has as potential parents all other on $(X, Y)$ RVs. Nevertheless, we want a single GCPF that determines the CPD for any ground $o n(X, Y) \mathrm{RV}$. This implies that such GCPFs have to be quite general. More precisely, a GCPF has to be able to deal with any set of potential parents. However, once we have learned such a GCPF for each probabilistic predicate, we can use it with any ordering of RVs. That means that if we decide to change the ordering, we do not have to relearn the GCPFs. This is different from the propositional case where CPDs are used that are much less general than GCPFs and where, if we change the ordering, some CPDs have to be relearned (because they use too many parents or not enough parents) (Teyssier and Koller 2005).

\subsubsection{Generalized ordering-search}

The above two differences between the propositional case and the relational case each lead to a modification to the original (propositional) ordering-search algorithm. First, we exploit the fact that GCPFs can be used with any ordering. In the original ordering-search, a search over orderings is performed and for each ordering (some of) the CPDs are relearned and applied to compute the score for that ordering. In our new algorithm we avoid this relearning of CPDs by learning general GCPFs once and then reusing them. Second, we need another modification to the original algorithm since in the relational case we do not want to learn an ordering on the RVs directly, but a model that determines an ordering given a domain of discourse.

The above modifications result in the following generalized ordering-search algorithm for learning directed probabilistic logical models. In the first step we learn a GCPF for each probabilistic predicate by sampling from all orderings. In the second step we learn a model of the optimal ordering by applying the learned GCPFs to various orderings. We discuss these two steps in more detail in the next sections.

\subsection{Step 1: learning generalized conditional probability functions (GCPFs)}

Formally we define a GCPF for a probabilistic predicate $p$ as a function $f$ such that for any set $\mathcal{R}$ of ground RVs, any target $T \in \mathcal{R}$ built from $p$, any assignment $v_{T}$ to $T$, any set $E \subseteq \mathcal{R}$ of known evidence RVs and any assignment $v_{E}$ to the RVs in $E, f\left(T, v_{T}, E, v_{E}, \mathcal{R}\right)=$ $P\left(T=v_{T} \mid \mathcal{R}, E=v_{E}\right)$, i.e. the probability that $T$ has value $v_{T}$ given the set of all RVs and the values of all RVs in $E$. 
A GCPF can be represented as a relational probability tree (Fierens et al. 2005b; Neville et al. 2003). A relational probability tree represents a probability distribution over the values of a target RV conditioned on the interpretation of some input predicates. Hence, to represent a GCPF by a relational probability tree we simply have to represent the "inputs" of the GCPF $\left(T, \mathcal{R}, E\right.$ and $v_{E}$ ) under the form of an interpretation (additionally, all logical predicates of the LBN are available as background knowledge to the GCPF as well). A test in an internal node of the relational probability tree can be any Prolog query about this interpretation. Concretely this means that tests can be about the target RV $T$, about existence of a certain $\mathrm{RV}$ or RVs (a test on $\mathcal{R}$ ), about whether an existing RV is known or unknown (a test on $E$ ) and about the value of an existing and known RV (a test on $v_{E}$ ).

Given an ordering on the set of RVs, the GCPFs determine not only the CPDs for all RVs but can at the same time be used to identify their parents. To determine the parents of a target RV $T$ built from $p$, the GCPF for $p$ is used with, as the set $E$, all potential parents of $T$ (all RVs preceding $T$ in the ordering). We then check which of these potential parents' values are effectively tested in the GCPF and these become the actual parents of $T$. Note that this assumes that the GCPFs are selective. For instance, probability trees are selective since they typically do not effectively test all the inputs but only the most relevant ones.

GCPFs under the form of relational probability trees can be learned by the TILDE system (Blockeel and De Raedt 1998) adapted to learn probability trees (Fierens et al. 2005b). For each probabilistic predicate $p$ we take a two-step approach. In the first step we build a training dataset for this GCPF, the GCPF dataset, by sampling from the original dataset. In the second step we apply TILDE on this GCPF dataset to learn a relational probability tree. We now discuss the first step in more detail.

In order to learn a GCPF for a probabilistic predicate $p$, we need a GCPF dataset containing labeled examples (the number of examples is a parameter of our algorithm). Each example consists of a set of RVs $\mathcal{R}$, a target RV $T$ built from $p$ and a set of known RVs $E$ with their real values $v_{E}$. The label of an example is the real value $v_{T}$ of $T$ for that example. Note that we want our GCPFs to be applicable to any possible ordering of RVs, which means that a given target RV $T$ can have any set of potential parents $E$. Hence, constructing an example for a GCPF dataset can be done as follows:

1. randomly select an example from the original dataset

2. randomly select a target RV $T$ from the set $\mathcal{R}$ of all RVs built from $p$ in this example

3. randomly select a subset of the set $\mathcal{R} \backslash\{T\}$ and call this set $E$

4. look up the values $v_{T}$ and $v_{E}$ of $T$ and $E$ in this example.

\subsection{Step 2: learning a model of the optimal ordering}

In the first step we learned a set of GCPFs. Since each GCPF needs as input the set of potential parents and this set depends on the ordering used, the second step consists of learning which ordering works best. Such an ordering together with the learned GCPFs fully determines an LBN. Since the precise set of RVs depends on the domain of discourse, we cannot simply learn a specific ordering. Instead we need to learn a general model that for any domain of discourse determines the optimal ordering on the corresponding set of ground RVs.

It is very complex to learn a model that directly predicts an ordering on an arbitrary set. Instead we take the simpler approach of learning a model that predicts for a given ordering and two given RVs adjacent in this ordering whether it is beneficial or not to swap these two RVs. Given such a model, we can determine the ordering for any set of RVs using an appropriate sorting procedure. Concretely, we start from the lexicographical ordering on the 
set of RVs and traverse the ordering, in each step comparing two adjacent RVs and swapping them if they are in the wrong order, until we reach the end.

To determine the optimal ordering we try to find the ordering that maximizes the likelihood of a training dataset. ${ }^{2}$ Concretely, we use a relational regression tree that takes as input an ordering and two RVs adjacent in this ordering and that predicts the gain in likelihood for swapping the two RVs (if the gain is positive, they will be swapped in the bubblesort, otherwise not). To learn such a regression tree, we take a two-step approach. In the first step we construct a regression dataset by computing differences in likelihood for various orderings using the previously learned GCPFs. In the second step we apply Tilde on this dataset to learn a relational regression tree (Blockeel and De Raedt 1998). We now discuss the first step in detail.

To construct the regression dataset we construct a number of examples (the number is user-specified) as follows:

1. randomly select an example from the original dataset

2. randomly select an ordering $O$ on the set of all RVs in this example

3. randomly select two RVs $R V_{1}$ and $R V_{2}$ adjacent in $O$

4. compute the likelihood for $O$ and for the ordering obtained by swapping $R V_{1}$ and $R V_{2}$ in $O$ (this step uses the learned GCPFs)

5. create an example consisting of an interpretation describing $R V_{1}, R V_{2}$, the set of RVs preceding $R V_{1}$ and $R V_{2}$ in $O$ and the set of RVs following $R V_{1}$ and $R V_{2}$ in $O$, and labeled with the gain in likelihood for swapping $R V_{1}$ and $R V_{2}$, as computed in step 4.

\subsection{Overview of generalized ordering-search}

For the sake of clarity, we now give a brief overview of the entire generalized orderingsearch algorithm.

1. For each probabilistic predicate $p$ :

- Construct a GCPF dataset $D_{p}^{g c p f}$

- Learn a probability tree $\mathcal{T}_{p}$ from $D_{p}^{g c p f}$

2. Learn a regression tree $\mathcal{T}^{\text {regr }}$ that specifies the optimal ordering for any pair of RVs:

- Construct a regression dataset $D^{r e g r}$ by traversing the space of orderings and computing the difference in likelihood between each two neighboring orderings (using the trees $\mathcal{T}_{p}$ )

- Learn a regression tree $\mathcal{T}^{\text {regr }}$ from $D_{p}^{\text {regr }}$

\subsection{Related work}

There are two existing algorithms for learning directed probabilistic logical models that are strongly related to generalized ordering-search: the learning algorithms for Probabilistic Relational Models (PRMs) and Bayesian Logic Programs (BLPs). Both algorithms are essentially upgrades of structure-search to the relational case but differ in how they avoid cyclic dependencies.

The learning algorithm for PRMs (Getoor et al. 2001) checks acyclicity of each candidate structure at the predicate level (with a structure we mean a set of conditional dependencies).

\footnotetext{
${ }^{2}$ In the experiments we always use another training dataset than the one used to learn GCPFs.
} 
To accommodate for dependencies that are cyclic at the predicate level but acyclic at the ground level (e.g. whether a person has a gene depends on whether his/her parents have the gene), PRMs let the user define guaranteed acyclic relationships (e.g. the parent relationship). While this approach is indeed sufficient for simple examples, it is rather restrictive for more general cases since it can become quite difficult for a user to specify all such relationships beforehand. Consider our blocks world example. We want to specify that an on $/ 2 \mathrm{RV}$ can depend on all other on $/ 2 \mathrm{RVs}$ preceding it in some ordering and we want to learn the optimal ordering. Obviously, an ordering needs to be acyclic. Hence, using PRMs the approach would be to predefine a number of ordering relations (in terms of auxiliary predicates), declare them as guaranteed acyclic and let the learning algorithm choose the best one. However, given a number of auxiliary predicates it can become quite cumbersome to enumerate all acyclic relationships that can be build out of them. Using our generalized ordering-search algorithm, this problem does not exist since our algorithm learns the optimal ordering directly.

The learning algorithm for BLPs (Kersting and De Raedt 2001) requires each candidate structure to be acyclic on all of the examples in the dataset. Concretely, BLPs compute the ground Bayesian network induced by the candidate structure on each example and test acyclicity of all these ground networks. While this approach is indeed less restrictive than that of PRMs, it can be very inefficient. Teyssier and Koller (2005) already reported for the propositional case that the acyclicity tests required for structure-search make it less efficient than ordering-search. For BLPs this problem is even magnified. Whereas in the propositional case each candidate structure requires only one acyclicity test with computational cost independent of the size of the data, in the case of BLPs each candidate structure requires an acyclicity test for each example. Hence, the computational cost of the acyclicity tests for a single candidate structure depends on the size of the data, both on the number of examples and on the size of a single example. In contrast, our generalized ordering-search algorithm does not require any acyclicity tests.

\section{Experiments}

\subsection{Setup}

In this section, we want to evaluate empirically how well our contributions perform in practice. In particular, we want to investigate the following questions:

Q1 How well can our method learn an ordering? We will compare with a "random" order (chosen arbitrarily) and with an "expert" order provided by a (somewhat) knowledgeable expert.

Q2 How does our method of learning a GCPF compare with a more classical approach of (iterating over) learning a conditional probability distribution for a particular ordering?

We will use several evaluation criteria to answer these questions:

C1 Accuracy: To evaluate the accuracy of the models, we compute the log-likelihood of a test set according to the different models learned on the training set. The closer to zero, the better the model is.

C2 Compactness: Besides accuracy of the models, complexity is also an important evaluation criterion. We will consider the size of the models as an indication for the complexity. In general, smaller models are preferred. 
C3 Interpretability: A related criterion is interpretability. We will try to (subjectively) evaluate the top of the learned decision trees.

C4 Efficiency: Finally, we want to evaluate what is the computational cost of our approach. For this, we provide timings of our experiments.

All the reported results show the average over 10 runs with the corresponding standard deviations. Unless mentioned otherwise, the testset for calculating the log-likelihoods contained 10000 examples. For all the experiments default parameters were chosen to learn the GCPFs (based on randomization tests) and the regression trees.

\subsection{Domains}

We first describe the different domains in which we evaluated our approach.

\subsubsection{Genetics domain}

As a first domain we use a classical simple genetics domain as described by Example 1 in Sect. 3.1. Given is a set of people, whether they are male or female, the parent relationship and for a number of persons whether they have a particular genetic property. To show generalization over different domains of discourse we opted to vary the size of the set of people between 4 and 24. The genetic property is assumed to be dominant: every person has two (unknown) chromosomes and if at least one of these has the property, then the person will have the property. The task is then to predict for every person in the family tree the probability that he/she will have the genetic property.

The predicate gene $/ 1$ is a probabilistic predicate and the logical predicates we used are mother $/ 2$, father $/ 2$ and parent $/ 2$. In this domain, an example is a family tree and $D^{g c p f}$, the size of the dataset used to learn the GCPFs, is the number of family trees. From these we randomly select examples with a probability of 0.10 .

\subsubsection{Blocks world domain}

Recently, there has been interest in learning probabilistic logical models for Relational Reinforcement Learning (RRL) (Croonenborghs et al. 2007). One often used testbed in RRL is the blocks world (Slaney and Thiébaux 2001). In the blocks world an agent can observe a state that consists of a number of stacks of blocks. Such a state is described by a set of on $/ 2$ facts that indicate which blocks are stacked onto each other. The agent can perform a move $(A, B)$ action that tries to move block $A$ onto block $B .^{3}$ If one of the two blocks has another block stacked on it, the action will have no result. In these experiments, the task is to learn the transition function for such a blocks world, i.e. the aim is to learn an LBN that can predict the probability distribution over possible next states given a certain state and action. The LBN for this domain consists of a probabilistic predicate on $/ 2$ and logical predicates on_previous $/ 2$ to describe the previous state and action $/ 1$ to model the action that is executed as described in Example 2 (Sect. 3.1).

The number of examples for $D^{g c p f}$ refers to the number of state transitions. As in the genetics domain, a sampling rate of 0.10 is used to select examples.

We will consider two variants of the blocks world where actions have stochastic effects. In both variations the number of blocks is varied between six and eight for different state transitions.

\footnotetext{
${ }^{3} B$ can also be the floor.
} 
Blocks world with earthquake risk In the first variant, an earthquake can occur after the normal effect of the action with a probability of 0.30 . After an earthquake, all blocks will be on the floor.

Blocks world with unstable blocks In the second variant of the blocks world, blocks are unstable. This means that after the normal effect of an action, every block has (independently) a probability of 0.15 of falling down. After a block falls down, this block and all blocks above it will be on the floor. This environment is interesting as there is an intuitive way to model the next state: a block is on the floor if it has fallen down itself or if any block which was below it is on the floor.

\subsubsection{Cora}

We consider a subset of the Cora dataset (McCallum et al. 1999). This dataset consists of a set of 682 papers and their references and has been used ${ }^{4}$ for various tasks, such as predicting the high-level topic of a paper (Neville et al. 2003). We consider the task of learning an LBN that models the dependencies between the topics of all papers (topics of two papers might be dependent when the papers are written by the same authors, when one paper cites the other, etc.). We use a probabilistic predicate topic/1 and logical predicates author $/ 2$, cites $/ 2$ and year $/ 2$ (and a several background knowledge predicates such as co-authorship, ... ).

\subsection{Accuracy}

In a first series of experiments, we compare the likelihood of the different models. In each case, we are considering an a priori defined "expert ordering", a "random ordering", and the ordering we learned as described in Sect. 3.4. The "expert ordering" is not always the optimal ordering, but the best ordering we can define a priori. In the genetics domain, the expert ordering is constructed in such a way that the RVs representing the genes of the parents come before those of their children. For the blocks world domain with earthquake risk, it is not straightforward to construct an optimal ordering for this domain, therefor we used the lexicographical order as "expert order". This way, RVs on $/ 2$ with the same block as their first arguments will be ordered together. As an expert ordering in the blocks world with unstable blocks, the on $/ 2$ atoms have as potential parents atoms where the second argument is at a lower height in the previous configuration. As a baseline to compare with, we also mention the likelihood of a model giving the prior distribution for every random variable (i.e. a model in which all variables are independent).

We will also investigate whether, given a particular ordering, it is better to use a general GCPF or an ordering-specific CPD. After learning the GCPF and the optimal ordering, we generate training examples for this fixed learned ordering, and learn a (specialized) CPD from it. This means that on the one hand the learned CPD will be less general, as it will not be able to predict any other conditional probability, but on the other hand it may be more accurate because there are more training examples available for the chosen network structure (while for the GCPF, the same number of examples cover any possible network structure). We also compare with a CPD learned from an a priori chosen ordering. Since the number of RVs varies, we use the lexicographical ordering for this. Note that for the genetics domain, this behaves as a random ordering and for the blocks world domains as an expert ordering. We will use the latter notations to refer to this fixed lexicographical ordering for learning CPDs.

\footnotetext{
${ }^{4}$ Several variants of the Cora dataset have been used in different papers, so results are difficult to compare.
} 
Table 1 Log-likelihoods of the models for the genetics domain

\begin{tabular}{|c|c|c|c|}
\hline Ordering & Size $D^{g c p f}$ & Size $D^{r e g r}$ & Log-likelihood \\
\hline random order & 200 & - & $-8.185 \pm 0.048$ \\
\hline expert order & 200 & - & $-8.146 \pm 0.068$ \\
\hline learned order & 200 & 100 & $-8.104 \pm 0.048$ \\
\hline learned order & 200 & 500 & $-8.044 \pm 0.095$ \\
\hline learned order & 200 & 1000 & $-8.102 \pm 0.050$ \\
\hline random order & 500 & - & $-7.474 \pm 0.085$ \\
\hline expert order & 500 & - & $-6.549 \pm 0.169$ \\
\hline learned order & 500 & 100 & $-7.166 \pm 0.091$ \\
\hline learned order & 500 & 500 & $-7.185 \pm 0.124$ \\
\hline learned order & 500 & 1000 & $-7.022 \pm 0.114$ \\
\hline random order & 1000 & - & $-7.331 \pm 0.026$ \\
\hline expert order & 1000 & - & $-6.391 \pm 0.044$ \\
\hline learned order & 1000 & 100 & $-6.985 \pm 0.090$ \\
\hline learned order & 1000 & 500 & $-6.892 \pm 0.093$ \\
\hline learned order & 1000 & 1000 & $-6.756 \pm 0.086$ \\
\hline random order & 5000 & - & $-7.173 \pm 0.022$ \\
\hline expert order & 5000 & - & $-6.142 \pm 0.022$ \\
\hline learned order & 5000 & 500 & $-6.738 \pm 0.105$ \\
\hline learned order & 5000 & 1000 & $-6.710 \pm 0.078$ \\
\hline random order & 10000 & - & $-7.181 \pm 0.012$ \\
\hline expert order & 10000 & - & $-6.103 \pm 0.016$ \\
\hline learned order & 10000 & 500 & $-6.623 \pm 0.114$ \\
\hline learned order & 10000 & 1000 & $-6.660 \pm 0.109$ \\
\hline prior model & - & - & $-8.956 \pm 0.160$ \\
\hline
\end{tabular}

Genetics domain Table 1 shows the results for the genetics domain for varying sizes of the GCPF training set. These results show that the learned ordering performs significantly better than a random order, but is outperformed by the optimal ordering. Also observe that for this domain a reasonably good ordering is learned with a small number of examples in $D^{r e g r}$.

The results of learning a CPD based on the learned ordering and the lexicographical ordering can be found in Table 2 for the genetics domain. The last two columns in this table repeat the results from Table 1 (without standard deviations). It shows that an ordering applied to the GCPF performs similar to directly learning a CPD for this ordering. As a result, the log-likelihoods obtained by the CPD from the learned ordering are better than those obtained by the CPD of the random (i.e. lexicographical) ordering.

Blocks world domain The likelihoods obtained by the GCPF for the blocks world with earthquake risk can be found in Table 3. As in the genetics domain, our learned ordering performs better than a random ordering. It also shows that the "expert ordering" only does better than the random ordering for bigger sizes of $D^{g c p f}$, but performs worse than the learned order for smaller dataset sizes. On the other hand, learning a good ordering is harder than in the genetics domain which is also shown by the relative big differences in log- 
Table 2 Log-likelihoods of the learned CPD models for the genetics domain

\begin{tabular}{|c|c|c|c|c|c|}
\hline \multirow{2}{*}{$\begin{array}{l}\text { Size } \\
D^{g c p f / c p d}\end{array}$} & \multirow{2}{*}{$\begin{array}{l}\text { Size } \\
D^{r e g r}\end{array}$} & \multicolumn{2}{|l|}{ CPD } & \multicolumn{2}{|l|}{ GCPF } \\
\hline & & Learned & Random & Learned & Random \\
\hline \multirow[t]{3}{*}{200} & 100 & $-8.134 \pm 0.085$ & $-8.119 \pm 0.023$ & -8.104 & -8.185 \\
\hline & 500 & $-8.098 \pm 0.071$ & & -8.044 & \\
\hline & 1000 & $-8.134 \pm 0.174$ & & -8.102 & \\
\hline \multirow[t]{3}{*}{500} & 100 & $-7.317 \pm 0.133$ & $-7.423 \pm 0.047$ & -7.166 & -7.331 \\
\hline & 500 & $-7.167 \pm 0.120$ & & -7.185 & \\
\hline & 1000 & $-6.920 \pm 0.105$ & & -7.022 & \\
\hline \multirow[t]{3}{*}{1000} & 100 & $-6.930 \pm 0.086$ & $-7.332 \pm 0.025$ & -6.985 & -7.331 \\
\hline & 500 & $-6.870 \pm 0.083$ & & -6.892 & \\
\hline & 1000 & $-6.839 \pm 0.135$ & & -6.756 & \\
\hline 5000 & 500 & $-6.655 \pm 0.079$ & $-7.275 \pm 0.096$ & -6.738 & -7.173 \\
\hline 10000 & 500 & $-6.640 \pm 0.104$ & $-7.158 \pm 0.019$ & -6.623 & -7.181 \\
\hline
\end{tabular}

likelihoods between different runs. Another observation is that sometimes using bigger sizes of $D^{r e g r}$ decreases performance which is probably due to overfitting.

Table 4 shows that in this domain directly learning a CPD from the "expert ordering" performs better than applying the expert ordering in the GCPF (there are more training examples specific for that ordering). This does however not hold for the learned ordering as applying the learned ordering on the GCPF performs better than directly learning a CPD from this ordering. Moreover, for small sizes of $D^{g c p f}$ an ordering can be learned that performs better than the expert ordering. (Note that it is easier to create bigger datasets for $\left.D^{r e g r}\right)$. In future work we plan to investigate the possibilities to iterate between learning a GCPF and learning an ordering. Such a strategy may avoid overfitting of the learned ordering and obtain a more accurate GCPF in the subspace of cases where it will be used together with the eventual ordering.

Table 5 shows the GCPF log-likelihoods and Table 6 the CPD log-likelihoods for the blocks world with unstable blocks. An interesting observation here is that although one expects the "expert ordering" to perform very well, the learned ordering does slightly better. They both are significantly better than the random order. The results for the CPD likelihoods are similar to the previous blocks world domain and therefore only a limited number of results are shown.

Cora dataset Finally, Table 7 shows the GCPF log-likelihoods for the Cora dataset. Here too, the GCPF is significantly better than the a priori model, whatever order is used. However, learning an order does not help much. As the "expert-order" we ordered the papers by publication date, assuming that references to earlier papers may give a hint at the subject of later papers. The learned ordering function validates this guess: in most of our experiments it contains only one test, either a published_before/2 or a paper_refers_to/2 test. The ordering function predicts only small likelihood differences, and this is confirmed by the results in Table 7.

Summary To summarize the accuracy results (see also Table 8): it is shown that the model using a learned ordering performs better than a random order except when a very small 
Table 3 Log-likelihoods of the GCPF models for the blocks world with earthquake risk

\begin{tabular}{|c|c|c|c|}
\hline Ordering & Size $D^{g c p f}$ & Size $D^{r e g r}$ & Log-likelihood \\
\hline random order & 200 & - & $-4.940 \pm 0.320$ \\
\hline expert order & 200 & - & $-5.225 \pm 0.278$ \\
\hline learned order & 200 & 100 & $-4.612 \pm 0.185$ \\
\hline learned order & 200 & 500 & $-4.993 \pm 0.172$ \\
\hline learned order & 200 & 1000 & $-5.055 \pm 0.304$ \\
\hline random order & 500 & - & $-3.859 \pm 0.116$ \\
\hline expert order & 500 & - & $-3.905 \pm 0.171$ \\
\hline learned order & 500 & 100 & $-3.906 \pm 0.360$ \\
\hline learned order & 500 & 500 & $-3.789 \pm 0.267$ \\
\hline learned order & 500 & 1000 & $-3.907 \pm 0.227$ \\
\hline random order & 1000 & - & $-2.980 \pm 0.136$ \\
\hline expert order & 1000 & - & $-3.085 \pm 0.199$ \\
\hline learned order & 1000 & 100 & $-2.604 \pm 0.418$ \\
\hline learned order & 1000 & 500 & $-2.539 \pm 0.114$ \\
\hline learned order & 1000 & 1000 & $-2.572 \pm 0.178$ \\
\hline random order & 5000 & - & $-1.714 \pm 0.097$ \\
\hline expert order & 5000 & - & $-1.475 \pm 0.120$ \\
\hline learned order & 5000 & 500 & $-1.416 \pm 0.514$ \\
\hline learned order & 5000 & 1000 & $-1.343 \pm 0.230$ \\
\hline random order & 10000 & - & $-1.578 \pm 0.027$ \\
\hline expert order & 10000 & - & $-1.429 \pm 0.038$ \\
\hline learned order & 10000 & 1000 & $-1.595 \pm 0.164$ \\
\hline prior model & - & - & $-21.194 \pm 0.0318$ \\
\hline
\end{tabular}

sample is used to learn the ordering. When comparing with the expert ordering, sometimes the learned ordering performs better, sometimes the expert ordering. Taking larger samples to create the training sets, both for learning the GCPF as for learning the ordering function, increases performance.

\subsection{Compactness}

In this section, we first examine more closely the CPDs learned for the random, expert and learned ordering from the point of view of the compactness of the learned theory. Table 9 shows the sizes (number of nodes of the decision trees) of these CPDs. One can see that the CPDs learned using a learned ordering are simpler than the CPDs learned using a random ordering, and comparable in size with the CPDs learned using an expert ordering.

Table 10 shows more details for the genetics domain with $\left|D^{\text {regr }}\right|=500\left(\left|D^{\text {regr }}\right|\right.$ is less important in this domain). In this domain, the CPD for the learned ordering is more compact than the one for the random ordering (certainly for smaller values of $D^{g c p f}$ ). Also note that the size of ordering function stays rather small, although it can be dependent on a specific GCPF.

As mentioned above, for the Cora dataset, ordering did not make much difference. The size of the learned GCPF was $19.3 \pm 5.1$ nodes. 
Table 4 Log-likelihoods of the learned CPD models for the blocks world with earthquake risk

\begin{tabular}{|c|c|c|c|c|c|}
\hline \multirow{2}{*}{$\begin{array}{l}\text { Size } \\
D^{g c p f / c p d}\end{array}$} & \multirow{2}{*}{$\begin{array}{l}\text { Size } \\
D^{r e g r}\end{array}$} & \multicolumn{2}{|l|}{ CPD } & \multicolumn{2}{|l|}{ GCPF } \\
\hline & & Learned & Expert & Learned & Expert \\
\hline \multirow[t]{3}{*}{200} & 100 & $-5.563 \pm 1.542$ & $-3.740 \pm 0.525$ & -4.612 & -5.225 \\
\hline & 500 & $-7.063 \pm 4.175$ & & -4.993 & \\
\hline & 1000 & $-4.365 \pm 0.914$ & & -5.055 & \\
\hline \multirow[t]{3}{*}{500} & 100 & $-4.342 \pm 0.691$ & $-3.418 \pm 0.128$ & -3.906 & -4.326 \\
\hline & 500 & $-4.860 \pm 0.674$ & & -3.789 & \\
\hline & 1000 & $-4.524 \pm 0.328$ & & -3.907 & \\
\hline \multirow[t]{3}{*}{1000} & 100 & $-2.366 \pm 0.623$ & $-2.307 \pm 0.116$ & -2.604 & -3.085 \\
\hline & 500 & $-6.984 \pm 2.705$ & & -2.539 & \\
\hline & 1000 & $-4.410 \pm 0.791$ & & -2.572 & \\
\hline 5000 & 1000 & $-3.982 \pm 1.364$ & $-1.263 \pm 0.031$ & -1.343 & -1.475 \\
\hline 10000 & 1000 & $-2.896 \pm 0.922$ & $-1.030 \pm 0.042$ & -1.595 & -1.429 \\
\hline
\end{tabular}

Table 5 Log-likelihoods of the models for the blocks world domain with unstable blocks

\begin{tabular}{lccc}
\hline Ordering & Size $D^{g c p f}$ & Size $D^{\text {regr }}$ & Log-likelihood \\
\hline random order & 500 & - & $-4.138 \pm 0.041$ \\
expert order & 500 & - & $-3.917 \pm 0.054$ \\
learned order & 500 & 100 & $-3.836 \pm 0.095$ \\
learned order & 500 & 500 & $-3.608 \pm 0.529$ \\
random order & 1000 & - & $-3.400 \pm 0.131$ \\
expert order & 1000 & - & $-3.188 \pm 0.033$ \\
learned order & 1000 & 100 & $-3.573 \pm 0.168$ \\
learned order & 1000 & 500 & $-3.213 \pm 0.128$ \\
learned order & 1000 & 1000 & $-3.054 \pm 0.285$ \\
random order & 5000 & - & $-2.778 \pm 0.039$ \\
expert order & 5000 & - & $-2.571 \pm 0.036$ \\
learned order & 5000 & 500 & $-2.432 \pm 0.037$ \\
learned order & 5000 & 1000 & $-2.459 \pm 0.056$ \\
random order & 10000 & - & $-2.432 \pm 0.031$ \\
expert order & 10000 & - & $-2.623 \pm 0.058$ \\
learned order & 10000 & 1000 & $-2.450 \pm 0.079$ \\
prior model & - & - & $-21.194 \pm 0.0318$ \\
\hline & & & \\
\hline
\end{tabular}

\subsection{Efficiency}

In Table 10 we compare the time for learning a GCPF with the time for learning a CPD for a fixed ordering in the blocks world with earthquake risk. All timings are in seconds. Similar timings were obtained for the other domains under consideration. Also, timings scale well when larger samples are generated for training. 
Table 6 Log-likelihoods of the learned CPD models for the blocks world with unstable blocks

\begin{tabular}{|c|c|c|c|c|c|}
\hline \multirow{2}{*}{$\begin{array}{l}\text { Size } \\
D^{g c p f / c p d}\end{array}$} & \multirow{2}{*}{$\begin{array}{l}\text { Size } \\
D^{r e g r}\end{array}$} & \multicolumn{2}{|l|}{ CPD } & \multicolumn{2}{|l|}{ GCPF } \\
\hline & & Learned & Expert & Learned & Expert \\
\hline \multirow[t]{3}{*}{1000} & 100 & $-4.677 \pm 1.215$ & $-2.501 \pm 0.311$ & -3.573 & -3.188 \\
\hline & 500 & $-3.833 \pm 0.554$ & & -3.213 & \\
\hline & 1000 & $-3.296 \pm 0.294$ & & -3.054 & \\
\hline
\end{tabular}

Table 7 Log-likelihoods of the GCPF for the Cora dataset

\begin{tabular}{lc}
\hline Model & Log-likelihood \\
\hline random order & $-517.2 \pm 0.1$ \\
expert order & $-515.7 \pm 0.1$ \\
learned order & $-515.7 \pm 0.1$ \\
a priori model & $-574.8 \pm 0.1$ \\
\hline
\end{tabular}

Table 8 Sign tests for the obtained log-likelihoods of the different orderings in the above datasets

\begin{tabular}{llll}
\hline Dataset & Learned/random & Learned/expert & Random/expert \\
\hline genetics & $5 / 0$ & $1 / 4$ & $0 / 5$ \\
blocks world with earthquakes & $3 / 2$ & $4 / 1$ & $3 / 2$ \\
blocks world with unstable blocks & $3 / 1$ & $4 / 0$ & $3 / 1$ \\
Cora & $1 / 0$ & tie & $0 / 1$ \\
total & $12 / 3$ & $9 / 5$ & $6 / 9$ \\
\hline
\end{tabular}

Table 9 Compactness of the different models with $\left|D^{g c p f}\right|=5000$ and $\left|D^{\text {regr }}\right|=1000$

\begin{tabular}{llll}
\hline Domain & \multicolumn{2}{l}{ Number of nodes } & \\
\cline { 2 - 3 } & Random & Expert & Learned \\
\hline Blocks (earthquake) & - & $27.250 \pm 0.908$ & $25.000 \pm 2.538$ \\
Blocks (unstable) & - & $21.000 \pm 1.030$ & $27.875 \pm 1.696$ \\
Genetics & $30.200 \pm 1.016$ & $9.231 \pm 1.712$ & $21.900 \pm 1.535$ \\
\hline
\end{tabular}

\subsection{Interpretability}

In many cases, the ordering learned in our experiments (and in particular the top tests of the decision tree) reflected some knowledge which is important for the domains and provides a good way to structure a network.

As an example, Fig. 1 shows the ordering function learned in the gene domain with both the size of $D^{g c p f}$ and $D^{r e g r}$ equal to 1000 . Note that in the genetics domain, the expert order performed significantly better than the learned ordering. This decision tree shows that in general it is better if the RVs representing genes of children come after the RVs representing the genes of the parents (as the genes of the parents are (causes and hence) predictive for the children). Note that this is similar to the expert ordering, the difference in performance 
Table 10 Compactness and efficiency for learning models in the genetics domain with $\left|D^{r e g r}=500\right|$

\begin{tabular}{|c|c|c|c|c|}
\hline$\left|D^{g c p f}\right|$ & Mode & Time & Nodes & Log-likelihood \\
\hline \multirow[t]{4}{*}{200} & GCPF & $0.802 \pm 0.052$ & $1.300 \pm 0.353$ & $-8.044 \pm 0.095$ \\
\hline & ordering function & $5.189 \pm 1.796$ & $1.600 \pm 0.864$ & - \\
\hline & learned CPD & $0.899 \pm 0.087$ & $1.200 \pm 0.263$ & $-8.098 \pm 0.071$ \\
\hline & random $\mathrm{CPD}$ & $0.866 \pm 0.140$ & $1.222 \pm 0.236$ & $-8.090 \pm 0.067$ \\
\hline \multirow[t]{4}{*}{400} & GCPF & $5.459 \pm 0.473$ & $3.600 \pm 0.233$ & $-7.032 \pm 0.121$ \\
\hline & ordering function & $12.734 \pm 1.554$ & $4.444 \pm 0.534$ & - \\
\hline & learned CPD & $4.547 \pm 0.288$ & $3.222 \pm 0.236$ & $-7.025 \pm 0.184$ \\
\hline & random $\mathrm{CPD}$ & $5.351 \pm 0.568$ & $3.700 \pm 0.225$ & $-7.532 \pm 0.096$ \\
\hline \multirow[t]{4}{*}{1000} & GCPF & $39.013 \pm 3.103$ & $6.500 \pm 0.724$ & $-6.892 \pm 0.093$ \\
\hline & ordering function & $21.284 \pm 3.718$ & $4.800 \pm 0.605$ & - \\
\hline & learned CPD & $33.142 \pm 2.667$ & $6.300 \pm 0.446$ & $-6.870 \pm 0.083$ \\
\hline & random $\mathrm{CPD}$ & $32.389 \pm 1.748$ & $6.300 \pm 0.446$ & $-7.332 \pm 0.017$ \\
\hline \multirow[t]{4}{*}{2000} & GCPF & $63.838 \pm 5.948$ & $9.900 \pm 0.675$ & $-7.054 \pm 0.062$ \\
\hline & ordering function & $8.898 \pm 1.624$ & $3.556 \pm 0.640$ & - \\
\hline & learned CPD & $47.606 \pm 2.000$ & $9.111 \pm 0.959$ & $-7.073 \pm 0.060$ \\
\hline & random CPD & $62.234 \pm 5.457$ & $13.000 \pm 1.414$ & $-7.329 \pm 0.037$ \\
\hline \multirow[t]{4}{*}{5000} & GCPF & $487.013 \pm 48.045$ & $21.400 \pm 1.157$ & $-6.738 \pm 0.105$ \\
\hline & ordering function & $33.102 \pm 2.162$ & $5.700 \pm 0.417$ & - \\
\hline & learned CPD & $534.395 \pm 64.672$ & $21.900 \pm 1.535$ & $-6.737 \pm 0.109$ \\
\hline & random $\mathrm{CPD}$ & $500.938 \pm 26.422$ & $20.200 \pm 1.016$ & $-7.169 \pm 0.017$ \\
\hline \multirow[t]{4}{*}{10000} & GCPF & $813.168 \pm 56.362$ & $35.900 \pm 2.856$ & $-6.623 \pm 0.114$ \\
\hline & ordering function & $17.516 \pm 1.212$ & $5.700 \pm 0.473$ & - \\
\hline & learned CPD & $829.347 \pm 57.709$ & $33.300 \pm 2.982$ & $-6.640 \pm 0.104$ \\
\hline & random $\mathrm{CPD}$ & $805.273 \pm 60.820$ & $32.300 \pm 1.707$ & $-7.158 \pm 0.019$ \\
\hline
\end{tabular}

with the expert ordering is probably due to the sorting method that is currently used in combination with small local effects such as illustrated in the rightmost node in Fig. 1.

As mentioned earlier, even though the ordering did not have a significant influence on the accuracy in the Cora dataset, the system consistently learned an ordering function that places earlier papers first and more recent papers at the end.

In summary, in both our synthetic and real-world domains, the learner was successful in reconstructing the direction of causal relationships between random variables.

\subsection{Conclusions}

Taking together the experimental results, we can answer our two questions as follows:

Q1 For the considered test domains, our method succeeds in learning an ordering that performs approximately equally well as the expert generated orderings w.r.t. accuracy, model complexity and as far as the language bias allows, also w.r.t. interpretability. The learned orderings are significantly better than random orderings.

Q2 Learning a GCPF, compared to a more classical approach of (iterating over) learning a conditional probability distribution for a particular ordering, produces a somewhat 
Table 11 Compactness and efficiency for learning models in the blocks world with earthquake risk with $\left|D^{r e g r}=1000\right|$

\begin{tabular}{|c|c|c|c|c|}
\hline$D^{g c p f} \mid$ & Mode & Time & Nodes & Log-likelihood \\
\hline \multirow[t]{4}{*}{200} & GCPF & $1.680 \pm 0.170$ & $3.400 \pm 0.233$ & $-5.055 \pm 0.304$ \\
\hline & ordering function & $266.942 \pm 41.641$ & $12.700 \pm 3.027$ & - \\
\hline & learned CPD & $1.848 \pm 0.147$ & $3.333 \pm 0.250$ & $-4.365 \pm 0.914$ \\
\hline & expert CPD & $1.780 \pm 0.148$ & $3.556 \pm 0.186$ & $1.460 \pm 0.169$ \\
\hline \multirow[t]{4}{*}{500} & GCPF & $5.412 \pm 0.415$ & $9.100 \pm 0.711$ & $-3.907 \pm 0.227$ \\
\hline & ordering function & $659.804 \pm 168.979$ & $33.300 \pm 4.098$ & - \\
\hline & learned CPD & $5.021 \pm 0.390$ & $7.000 \pm 0.544$ & $-4.524 \pm 0.328$ \\
\hline & expert CPD & $4.501 \pm 0.187$ & $7.700 \pm 0.473$ & $-3.418 \pm 0.128$ \\
\hline \multirow[t]{4}{*}{1000} & GCPF & $8.346 \pm 0.113$ & $14.800 \pm 0.492$ & $-2.572 \pm 0.178$ \\
\hline & ordering function & $470.531 \pm 193$ & $60.1 \pm 10.787$ & - \\
\hline & learned CPD & $8.550 \pm 0.988$ & $13.200 \pm 0.843$ & $-4.410 \pm 0.791$ \\
\hline & expert CPD & $7.900 \pm 0.273$ & $13.900 \pm 0.576$ & $-2.140 \pm 0.095$ \\
\hline \multirow[t]{4}{*}{2000} & GCPF & $17.797 \pm 0.583$ & $21.000 \pm 1.871$ & $-1.884 \pm 0.213$ \\
\hline & ordering function & $372 \pm 57.7$ & $51.000 \pm 4.950$ & - \\
\hline & learned CPD & $33.8 \pm 12.4$ & $15.667 \pm 3.629$ & $-2.047 \pm 0.527$ \\
\hline & expert CPD & $17.913 \pm 1.150$ & $19.333 \pm 1.472$ & $-1.390 \pm 0.074$ \\
\hline \multirow[t]{4}{*}{5000} & GCPF & $292.707 \pm 2.668$ & $38.143 \pm 1.771$ & $-1.343 \pm 0.230$ \\
\hline & ordering function & $832.574 \pm 370.131$ & $75.714 \pm 15.680$ & - \\
\hline & learned CPD & $305.184 \pm 17.595$ & $26.600 \pm 4.522$ & $-3.982 \pm 1.364$ \\
\hline & expert CPD & $286.274 \pm 2.273$ & $27.250 \pm 1.908$ & $-1.263 \pm 0.031$ \\
\hline \multirow[t]{4}{*}{10000} & GCPF & $762.232 \pm 24.852$ & $55.600 \pm 1.239$ & $-1.595 \pm 0.164$ \\
\hline & ordering function & $3971.604 \pm 1737.964$ & $58.700 \pm 13.726$ & - \\
\hline & learned CPD & $929.314 \pm 100.562$ & $33.400 \pm 5.602$ & $-2.896 \pm 0.922$ \\
\hline & expert CPD & $748.939 \pm 21.360$ & $47.200 \pm 1.897$ & $-1.030 \pm 0.04$ \\
\hline
\end{tabular}

larger but more general conditional probability function while the computational complexity of learning a GCPF is not larger than iterating a few times over learning a CPD for different orderings.

\section{Conclusions and further work}

In this paper we presented a novel algorithm for learning the structure of directed probabilistic logical models. This approach can be seen as a generalization of ordering search. Due to the generalizing power of relational decision tree learners, our approach can avoid the usual iteration over different candidate structures or orderings by following a two-phase learning process. We have implemented our algorithm and presented promising empirical results.

There are several directions for further work. First, we would like to evaluate our algorithm on larger real-world datasets with multiple probabilistic predicates since in the present paper we considered only datasets with one probabilistic predicate and we focused on how to model recursive dependencies for this predicate. A second direction for further work is 


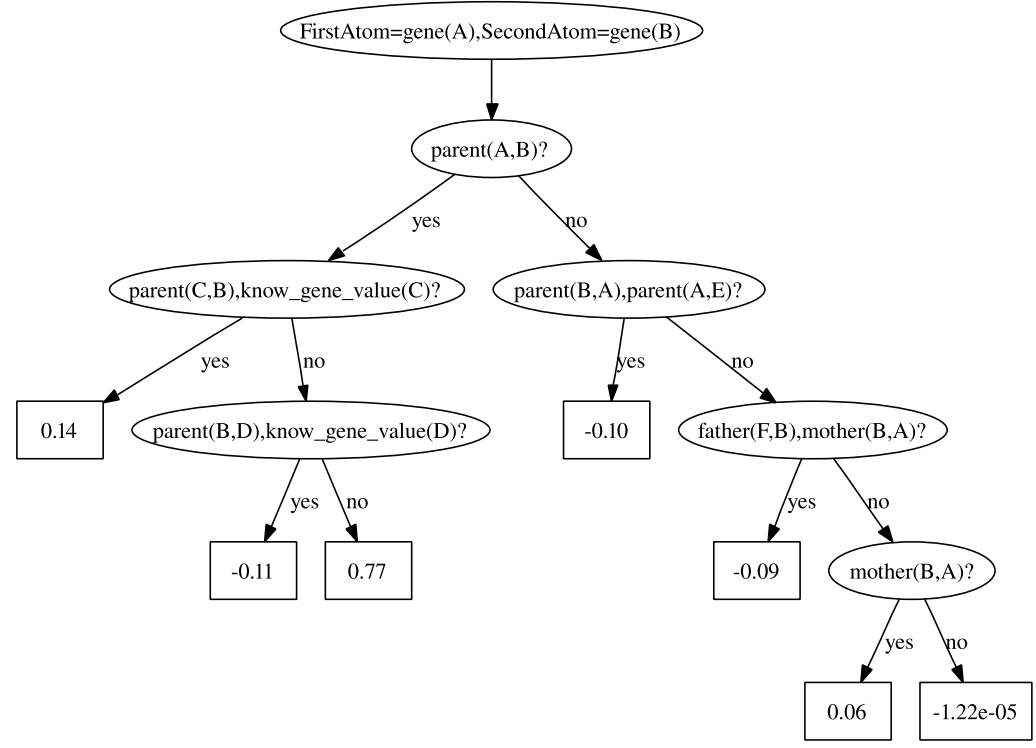

Fig. 1 Regression tree $\mathcal{T}^{\text {regr }}$ learned in the genetics domain with $\left|D^{g c p f}\right|=\left|D^{\text {regr }}\right|=1000$

the development of a more general representation of the model of the optimal ordering. Currently we use a regression tree that specifies for each two RVs which RV should be first in the ordering. This is a rather local model. It is interesting to investigate whether an ordering can be specified in a more global way. This would allow us to avoid the use of bubblesort to determine a concrete ordering and could be beneficial since in our current approach the result of the bubblesort depends on the initial (lexicographical) ordering. A third direction of future work is the investigation of more iterative methods where after learning a GCPF and an ordering, the ordering is used to generate training examples that are more interesting than the original random ones.

Acknowledgement Jan Ramon and Hendrik Blockeel are post-doctoral fellows of the Fund for Scientific Research of Flanders (FWO-Vlaanderen). Tom Croonenborghs and Daan Fierens are supported by the Institute for the Promotion of Innovation by Science and Technology in Flanders (IWT Vlaanderen).

\section{References}

Blockeel, H., \& De Raedt, L. (1998). Top-down induction of first order logical decision trees. Artificial Intelligence, 101(1-2), 285-297.

Croonenborghs, T., Ramon, J., Blockeel, H., \& Bruynooghe, M. (2007). Online learning and exploiting relational models in reinforcement learning. In M. Veloso (Ed.), Proceedings of the 20th international joint conference on artificial intelligence (pp. 726-731), Hyderabad, India. Menlo Park: AAAI Press.

De Raedt, L., \& Kersting, K. (2004). Probabilistic inductive logic programming. In Lecture notes in computer science: Vol. 3244. Proceedings of the 15th international conference on algorithmic learning theory (pp. 19-36). Berlin: Springer.

Fierens, D., Blockeel, H., Bruynooghe, M., \& Ramon, J. (2005a). Logical Bayesian networks and their relation to other probabilistic logical models. In Lecture notes in computer science: Vol. 3625. Proceedings of the 15th international conference on inductive logic programming (pp. 121-135). Berlin: Springer.

Fierens, D., Ramon, J., Blockeel, H., \& Bruynooghe, M. (2005b). A comparison of approaches for learning first-order logical probability estimation trees. In 15th international conference on inductive logic 
programming, late-breaking papers (pp. 11-16). Technische Universität München. (Technical Report TUM-I0510, Technische Universität München).

Getoor, L., Friedman, N., Koller, D., \& Pfeffer, A. (2001). Learning probabilistic relational models. In S. Dzeroski \& N. Lavrac (Eds.), Relational data mining (pp. 307-334). Berlin: Springer.

Heckerman, D., Geiger, D., \& Chickering, D. (1995). Learning Bayesian networks: the combination of knowledge and statistical data. Machine Learning, 20, 197-243.

Kersting, K., De Raedt, L. (2001). Towards combining inductive logic programming and Bayesian networks. In C. Rouveirol \& M. Sebag (Eds.), Lecture notes in computer science: Vol. 2157. Proceedings of the 11th international conference on inductive logic programming (pp. 118-131). Berlin: Springer.

McCallum, A., Nigam, K., \& Rennie, J., Seymore, K. (1999). A machine learning approach to building domain-specific search engines. In Proceedings of the 16th international joint conference on artificial intelligence (pp. 662-667). Los Altos: Kaufmann.

Neapolitan, R. (2003). Learning Bayesian networks. Prentice Hall: Upper Saddle River.

Neville, J., \& Jensen, D. (2004). Dependency networks for relational data. In Proceedings of the 4th IEEE international conference on data mining. Los Alamitos: IEEE Computer Society.

Neville, J., Jensen, D., Friedland, L., \& Hay, M. (2003). Learning relational probability trees. In Proceedings of the 9th ACM SIGKDD international, conference on knowledge discovery and data mining. New York: ACM.

Richardson, M., \& Domingos, P. (2006). Markov logic networks. Machine Learning, 62(1-2), 107-136.

Slaney, J., \& Thiébaux, S. (2001). Blocks world revisited. Artificial Intelligence, 125(1-2), 119-153.

Teyssier, M., \& Koller, D. (2005). Ordering-based search: a simple and effective algorithm for learning Bayesian networks. In Proceedings of the 21st conference on uncertainty in AI (pp. 584-590). Edinburgh: AUAI Press. 